\title{
Convergence of a Randomised Change Point Estimator in GARCH Models
}

\author{
George Awiakye-Marfo', Joseph Mung'atu², Patrick Weke ${ }^{3}$ \\ ${ }^{1}$ Department of Mathematics and Statistics, Pan African University, Nairobi, Kenya \\ ${ }^{2}$ Department of Statistics and Actuarial Science, JKUAT, Nairobi, Kenya \\ ${ }^{3}$ School of Mathematics, University of Nairobi, Nairobi, Kenya \\ Email: g.amarfo@aims.edu.gh
}

How to cite this paper: Awiakye-Marfo, G., Mung'atu, J. and Weke, P. (2021) Convergence of a Randomised Change Point Estimator in GARCH Models. Journal of Mathematical Finance, 11, 234-245. https://doi.org/10.4236/jmf.2021.112013

Received: February 7, 2021

Accepted: May 9, 2021

Published: May 12, 2021

Copyright () 2021 by author(s) and Scientific Research Publishing Inc. This work is licensed under the Creative Commons Attribution International License (CC BY 4.0).

http://creativecommons.org/licenses/by/4.0/

\begin{abstract}
In this paper, the randomised pseudolikelihood ratio change point estimator for GARCH models in [1] is employed and its limiting distribution is derived as the supremum of a standard Brownian bridge. Data analysis to validate the estimator is carried out using the United states dollar (USD)-Ghana cedi (GHS) daily exchange rate data. The randomised estimator is able to detect and estimate a single change in the variance structure of the data and provides a reference point for historic data analysis.
\end{abstract}

\section{Keywords}

GARCH, Randomised, Limiting Distribution, Brownian Bridge, Volatility, CUSUM, IGARCH, Supremum

\section{Introduction}

Volatility models are becoming increasingly important due to their role in asset pricing and risk management. It is however, not directly observed and hence needs to be estimated. Since the introduction of Autoregressive conditional Heteroscedacisity $(\mathrm{ARCH})$ by [2] and its generalisation, Generalised $\mathrm{ARCH}$ (GARCH) by [3], these models have arguably been the most popular and used financial volatility models. It is, however, possible that structural changes such as "shocks" as a result of changes in institutions, financial crises, may cause the data generating parameters of these models to change. Failure to accommodate these parameter changes in the conditional and unconditional volatility of a series in the model may have serious impacts on the forecasting abilities of these models. In fact [4] and [5] showed that neglecting parameter changes in the application of $\operatorname{GARCH}(1,1)$ model to long time economic processes often lead to 
high persistence and for this reason, the IGARCH model of [6] was introduced. For a comprehensive study on change point problems, we refer to [7]. In GARCH models, the problems of change point estimation have been studied by various authors after the variance change test by [8] for independent observations. Some of the earliest work on GARCH change point estimation was by [9] based on the cumulative sum of squares of [8], and also the standardised residual based test of [10] with the aim of reducing large size distortions and low power of the CUSUM test of [9]. The authors showed that the proposed test statistic has a limiting distribution as the sup of a standard Brownian bridge via the invariance principle for mixingale sequences whilst in [11] the point processes theory was utilized to obtain a weak convergence limit of a model order change point process for GARCH models. The authors of [12] considered the problem of testing for parameter change in nonlinear time series with GARCH errors and showed that under some regularity assumptions, the limiting null distribution is a supremum of a standard Brownian Bridge. The authors of [13] proposed a weighted CUSUM test statistic to test for mean change in an $\mathrm{AR}(\mathrm{p})$ process and its limiting distribution was obtained via the mixing conditions for linear processes. In [14] estimates-based CUSUM change point test in ARMA-GARCH and non linear Autoregressive Conditional Duration (ACD) models were studied and the result of the quasi-maximum likelihood estimator (QMLE) from ACD models was used to derive the limiting null distribution of the CUSUM test. Later [15] considered a modified residual-based CUSUM test for location-scale time series models with heteroscedasticity and in both studies, the authors derived the limiting distribution derived as the supremum of a Brownian bridge.

The change point problems for GARCH models in literature have usually been viewed as deterministic. However, in [1], a randomised change point test for GARCH models and its consistency were derived. In this paper, the limiting distribution of the randomised estimator of [1] is derived and validated via the United States dollar (USD)-Ghana cedi (GHS) exchange rate data. The idea of the randomised estimator is to weigh down excessively large observations and hence obtain test statistic values different from the described deterministic cases. Identifying discontinuities helps improve the forecasting abilities of GARCH models.

This paper is organised into five sections. Section 1 is the Introduction, research methodology is presented in Section 2. In Section 3, the limiting distribution of the estimator is derived. Section 4 presents results and discussions whilst Section 5 concludes the study.

\section{Methodology}

Consider the model

$$
X_{t}=g\left(X_{t-1}, X_{t-2}, \cdots, X_{t-p} ; \theta_{t}\right)+\varepsilon_{t}
$$

where the errors $\varepsilon_{t}=\sigma_{t} z_{t}, \quad z_{t}$ has zero expectation and finite variance, $\sigma_{t}$ 
follows a GARCH (p,q) model, for instance a standard GARCH model. The conditional mean, $g\left(X_{t-1}, X_{t-2}, \cdots, X_{t-p} ; \theta_{t}\right)$ follows an autoregressive function. The test statistic for the change point is constructed by employing the likelihood ratio and derived as follows;

$$
-2 \log \Delta_{k}=n \log \hat{\sigma}_{n}^{2}-k \log \hat{\sigma}_{k}^{2}-(n-k) \log \hat{\sigma}_{k^{*}}^{2}
$$

consider $\hat{\sigma}_{k^{*}}^{2}=\hat{\sigma}_{k}^{2}+\delta$ and under the null hypothesis take $\delta \rightarrow 0$ as $n \rightarrow \infty$, we have

$$
-\frac{2}{n} \log \Delta_{k}=\log \left(\frac{\hat{\sigma}_{n}^{2}}{\hat{\sigma}_{k}^{2}}\right)=\log \left(1+\frac{\hat{\sigma}_{n}^{2}-\hat{\sigma}_{k}^{2}}{\hat{\sigma}_{k}^{2}}\right)=\log \left(1+\frac{H_{k}}{\hat{\sigma}_{k}^{2}}\right)
$$

where the variance estimates are given as

$$
\begin{gathered}
\hat{\sigma}_{n}^{2}(h)=\frac{1}{\sum_{t=1}^{n} h\left(X_{t}\right)} \sum_{t=1}^{n} h\left(X_{t}\right)\left(X_{t}-\hat{\theta}_{n}(h)\right)^{2}, \\
\hat{\sigma}_{k^{*}}^{2}(h)=\frac{1}{\sum_{t=k+1}^{n} h\left(X_{t}\right)} \sum_{t=k+1}^{n} h\left(X_{t}\right)\left(X_{t}-\hat{\theta}_{k^{*}}(h)\right)^{2}, \\
\hat{\sigma}_{k}^{2}(h)=\frac{1}{\sum_{t=1}^{n} h\left(X_{t}\right)}\left(\sum_{t=1}^{k} h\left(X_{t}\right)\left(X_{t}-\hat{\theta}_{n}(h)\right)^{2}+\sum_{t=k+1}^{n} h\left(X_{t}\right)\left(X_{t}-\hat{\theta}_{k^{*}}(h)\right)^{2}\right) .
\end{gathered}
$$

A detailed simplification of Equation (2), and an expression for $H_{k}$ can be found in [1]. The modified weighted test statistic is of the form;

$$
\tilde{e}_{n}=\max _{0<k<n}\left(\frac{\left(\sum_{t=1}^{n} h_{t}\right)^{2}}{\sum_{t=1}^{k} h_{t}\left(\sum_{t=1}^{n} h_{t}-\sum_{t=1}^{k} h_{t}\right)}\right)^{v} \frac{1}{\sqrt{n}}\left|S(k)-\left(\frac{\sum_{t=1}^{k} h_{t}}{\sum_{t=1}^{n} h_{t}}\right) S(n)\right|
$$

with $v \in(0,1 / 2)$ where $S(k)=\sum_{t=1}^{k} h_{t} X_{t}$ and $S(n)=\sum_{t=1}^{n} h_{t} X_{t}$

Finally, the test statistic as given in [1] is

$$
\tilde{e}_{n}=\max _{1 \leq k<n}\left(\frac{\left(\sum_{t=1}^{n} h_{t}\right)^{2}}{\sum_{t=1}^{k} h_{t}\left(\sum_{t=1}^{n} h_{t}-\sum_{t=1}^{k} h_{t}\right)}\right)^{v} \frac{1}{\sqrt{n}}\left|\sum_{t=1}^{k} h_{t} \varepsilon_{t}^{2}-\left(\frac{\sum_{t=1}^{k} h_{t}}{\sum_{t=1}^{n} h_{t}}\right) \sum_{t=1}^{n} h_{t} \varepsilon_{t}^{2}\right|
$$

with $v \in(0,1 / 2)$ and the estimator given as $\hat{k}=\arg \max _{1 \leq k<n} \tilde{e}_{k}$.

\section{Limiting Distribution of the Estimator}

Here we show the limiting distribution of the randomised estimator as described in Equation (4). We note from [16] that the GARCH model, $\sigma_{t}^{2}$ is an $\alpha$-mixing process, so is $h_{t}$ and hence $h_{t} \sigma_{t}^{2}$ by hereditary.

Assumption 1. The functions $h_{t}=g\left(X_{t-1}, X_{t-2}, \cdots, X_{t-p}, X_{t+1}, X_{t+2}, \cdots, X_{t+p}\right)$ and $g\left(X_{1}, X_{2}, \cdots, X_{2 p}\right)$ are real and positive functions on $\mathbb{R}^{2 p}$ such that $\mathbb{E}\left\{\left(1+\left\|X_{t}\right\|^{2+\alpha}\right)\left(h_{t}+h_{t}^{2+\alpha}\right)\right\}<\infty$ for $\alpha>0$.

We note that since $\varepsilon_{t}^{2}$ is not directly observable we replace with $\hat{\varepsilon}_{t}^{2}$ where $\hat{\varepsilon}_{t}^{2}=\varepsilon_{t}^{2}+\Lambda_{t}$ and $\Lambda_{t}=o_{P}(1)$ has been established in [12]. 
Proposition 1. If assumption (1) holds then under the null hypothesis of no change in variance we have $\tilde{e}_{n} \stackrel{w}{\longrightarrow} \frac{\sigma_{1} B^{0}(\tau)}{(\tau(1-\tau))^{v}}-\frac{\sigma_{2} \mathbb{E}\left(h_{t} \varepsilon_{t}^{2}\right)}{\mathbb{E}\left(h_{t}\right)^{v+1}(\tau(1-\tau))^{v}} W^{0}(\tau)$ as $n \rightarrow \infty$ where $\stackrel{w}{\longrightarrow}$ defines the weak convergence of the process, $\sigma_{1}^{2}=\sum_{t=-\infty}^{+\infty} \operatorname{Cov}\left(h_{0} \varepsilon_{0}, h_{t} \varepsilon_{t}\right) \neq 0$ and $\sigma_{2}^{2}=\sum_{t=-\infty}^{+\infty} \operatorname{Cov}\left(h_{0}, h_{t}\right) \neq 0 . \quad B^{0}(\tau)$ and $W^{0}(\tau)$ denote standard brownian motions on $[0,1]$.

Proof. Considering Equation (4) we make the following representation;

$$
\begin{gathered}
A_{k}=\left(\frac{\sum_{t=1}^{k} h\left(X_{t}\right)}{\sum_{t=1}^{n} h\left(X_{t}\right)}\right)^{-v}\left(\frac{\sum_{t=k+1}^{n} h\left(X_{t}\right)}{\sum_{t=1}^{n} h\left(X_{t}\right)}\right)^{-v} \text { and } \\
B_{k}=\left|\frac{\sum_{t=1}^{k} h\left(X_{t}\right) \varepsilon_{t}^{2}}{\left(\sum_{t=1}^{n} h\left(X_{t}\right)\right)^{v}}-\frac{\sum_{t=1}^{k} h\left(X_{t}\right)}{\sum_{t=1}^{n} h\left(X_{t}\right)} \frac{\sum_{t=1}^{n} h\left(X_{t}\right) \varepsilon_{t}^{2}}{\left(\sum_{t=1}^{n} h\left(X_{t}\right)\right)^{v}}\right|
\end{gathered}
$$

We re-write

$$
\begin{aligned}
B_{k}= & \frac{\frac{1}{n^{v}} \sum_{t=1}^{k}\left(h_{t} \varepsilon_{t}^{2}-\mathbb{E}\left(h_{t} \varepsilon_{t}^{2}\right)\right)}{\left(\frac{1}{n} \sum_{t=1}^{n} h_{t}\right)^{v}} \\
& -\frac{\sum_{t=1}^{k} h\left(X_{t}\right)}{\sum_{t=1}^{n} h\left(X_{t}\right)}\left|\frac{\sum_{t=1}^{n} h\left(X_{t}\right) \varepsilon_{t}^{2}}{\left(\sum_{t=1}^{n} h\left(X_{t}\right)\right)^{v}}-\frac{\sum_{t=1}^{n} h\left(X_{t}\right)}{\sum_{t=1}^{k} h\left(X_{t}\right)} \frac{\sum_{t=1}^{k} h\left(X_{t}\right) \varepsilon_{t}^{2}}{\left(\sum_{t=1}^{n} h\left(X_{t}\right)\right)^{v}}\right| \\
:= & L_{1}-L_{2}
\end{aligned}
$$

We consider $L_{2}$ as follows

$$
\begin{aligned}
L_{2}= & \frac{\sum_{t=1}^{k} h\left(X_{t}\right)}{\sum_{t=1}^{n} h\left(X_{t}\right)}\left(\frac{\frac{1}{n^{v}} \sum_{t=1}^{k}\left(h_{t} \varepsilon_{t}^{2}-\mathbb{E}\left(h_{t} \varepsilon_{t}^{2}\right)\right)}{\left(\frac{1}{n} \sum_{t=1}^{n} h_{t}\right)^{v}}\right. \\
& \left.+\frac{\frac{n}{n^{v}} \mathbb{E}\left(h_{t} \varepsilon_{t}^{2}\right)}{\left(\frac{1}{n} \sum_{t=1}^{n} h_{t}\right)^{v}}-\frac{\sum_{t=1}^{n} h\left(X_{t}\right)}{\sum_{t=1}^{k} h\left(X_{t}\right)} \frac{\sum_{t=1}^{k} h\left(X_{t}\right) \varepsilon_{t}^{2}}{\left(\sum_{t=1}^{n} h\left(X_{t}\right)\right)^{v}}\right) \\
= & \left(\frac{\sum_{t=1}^{k} h\left(X_{t}\right)}{\sum_{t=1}^{n} h\left(X_{t}\right)}\right)\left(\hat{L}_{1}+\hat{L}_{2}\right)
\end{aligned}
$$

For $\hat{L}_{2}$ we have

$$
\begin{aligned}
\hat{L}_{2} & =\frac{n \mathbb{E}\left(h_{t} \varepsilon_{t}^{2}\right)}{n^{v}\left(\frac{1}{n} \sum_{t=1}^{n} h_{t}\right)^{v}}-\frac{\sum_{t=1}^{n} h\left(X_{t}\right)}{\sum_{t=1}^{k} h\left(X_{t}\right)} \frac{\sum_{t=1}^{k} h\left(X_{t}\right) \varepsilon_{t}^{2}}{n^{v}\left(\frac{1}{n} \sum_{t=1}^{n} h_{t}\right)^{v}} \\
& =\frac{\mathbb{E}\left(h_{t} \varepsilon_{t}^{2}\right)}{\left(\frac{1}{n} \sum_{t=1}^{n} h_{t}\right)^{v}}\left(\frac{n}{n^{v}}-\frac{\sum_{t=1}^{n} h\left(X_{t}\right)}{\frac{1}{k} \sum_{t=1}^{k} h\left(X_{t}\right) n^{v}}\right)
\end{aligned}
$$




$$
\begin{aligned}
& =\frac{\mathbb{E}\left(h_{t} \varepsilon_{t}^{2}\right)}{\left(\frac{1}{n} \sum_{t=1}^{n} h_{t}\right)^{v}}\left(\frac{\frac{1}{n^{v}}\left(\frac{n}{k} \sum_{t=1}^{k} h_{t}-\sum_{t=1}^{n} h_{t}\right)}{\frac{1}{k} \sum_{t=1}^{k} h_{t}}\right) \\
& =\frac{\mathbb{E}\left(h_{t} \varepsilon_{t}^{2}\right)}{\tau\left(\frac{1}{n} \sum_{t=1}^{n} h_{t}\right)^{v}}\left(\frac{\frac{1}{n^{v}} \sum_{t=1}^{k}\left(h_{t}-\mathbb{E} h_{t}+\mathbb{E} h_{t}\right)-\frac{\tau}{n^{v}} \sum_{t=1}^{n}\left(h_{t}-\mathbb{E} h_{t}+\mathbb{E} h_{t}\right)}{\frac{1}{k} \sum_{t=1}^{k} h_{t}}\right)
\end{aligned}
$$

where $\tau=\frac{k}{n}$ hence Equation (5) becomes

$$
\hat{L}_{2}=\frac{\mathbb{E}\left(h_{t} \varepsilon_{t}^{2}\right)}{\tau\left(\frac{1}{n} \sum_{t=1}^{n} h_{t}\right)^{v}}\left(\frac{\frac{1}{n^{v}} \sum_{t=1}^{k}\left(h_{t}-\mathbb{E} h_{t}\right)-\frac{\tau}{n^{v}} \sum_{t=1}^{n}\left(h_{t}-\mathbb{E} h_{t}\right)}{\frac{1}{k} \sum_{t=1}^{k} h_{t}}\right)
$$

Consequently we have

$$
\begin{aligned}
L_{2}=\tau\left[\frac{\frac{1}{n^{v}} \sum_{t=1}^{k}\left(h_{t} \varepsilon_{t}^{2}-\mathbb{E}\left(h_{t} \varepsilon_{t}^{2}\right)\right)}{\left(\frac{1}{n} \sum_{t=1}^{n} h_{t}\right)^{v}}\right. \\
\left.+\frac{\mathbb{E}\left(h_{t} \varepsilon_{t}^{2}\right)}{\tau\left(\frac{1}{n} \sum_{t=1}^{n} h_{t}\right)^{v}}\left(\frac{\frac{1}{n^{v}} \sum_{t=1}^{k}\left(h_{t}-\mathbb{E} h_{t}\right)-\frac{\tau}{n^{v}} \sum_{t=1}^{n}\left(h_{t}-\mathbb{E} h_{t}\right)}{\frac{1}{k} \sum_{t=1}^{k} h_{t}}\right)\right]
\end{aligned}
$$

From Equation (4) we have

$$
\begin{aligned}
\tilde{e}_{n}= & \left(\frac{\sum_{t=1}^{k} h\left(X_{t}\right)}{\sum_{t=1}^{n} h\left(X_{t}\right)}\right)^{-v}\left(\frac{\sum_{t=k+1}^{n} h\left(X_{t}\right)}{\sum_{t=1}^{n} h\left(X_{t}\right)}\right)^{-v}\left(\frac{1}{n} \sum_{t=1}^{k} h_{t}\right)^{v} \\
& \times\left[\frac{\frac{1}{\sqrt{n}} \sum_{t=1}^{k}\left(h_{t} \varepsilon_{t}^{2}-\mathbb{E}\left(h_{t} \varepsilon_{t}^{2}\right)\right)}{\left(\frac{1}{n} \sum_{t=1}^{n} h_{t}\right)^{v}}\right]-\tau\left(\frac{\frac{1}{\sqrt{n}} \sum_{t=1}^{k}\left(h_{t} \varepsilon_{t}^{2}-\mathbb{E}\left(h_{t} \varepsilon_{t}^{2}\right)\right)}{\left(\frac{1}{n} \sum_{t=1}^{n} h_{t}\right)^{v}}\right. \\
& \left.+\frac{\mathbb{E}\left(h_{t} \varepsilon_{t}^{2}\right)}{\tau\left(\frac{1}{n} \sum_{t=1}^{n} h_{t}\right)^{v}}\left(\frac{\left.\frac{1}{\sqrt{n}} \sum_{t=1}^{k}\left(h_{t}-\mathbb{E} h_{t}\right)-\frac{\tau}{\sqrt{n}} \sum_{t=1}^{n}\left(h_{t}-\mathbb{E} h_{t}\right)\right)}{\frac{1}{k} \sum_{t=1}^{k} h_{t}}\right)\right)
\end{aligned}
$$

We evaluate the limit of the function $A_{k}$ as

$$
\left(\frac{\sum_{t=1}^{k} h\left(X_{t}\right)}{\sum_{t=1}^{n} h\left(X_{t}\right)}\right)^{-v}\left(\frac{\sum_{t=k+1}^{n} h\left(X_{t}\right)}{\sum_{t=1}^{n} h\left(X_{t}\right)}\right)^{-v}\left(\frac{1}{n} \sum_{t=1}^{n} h_{t}\right)^{v} \stackrel{w}{\longrightarrow} \frac{\mathbb{E}\left(h_{t}\right)^{v}}{(\tau(1-\tau))^{v}}
$$

Also from $L_{1}$ and $L_{2}$ we have

$$
L_{1} \stackrel{w}{\longrightarrow} \frac{\sigma_{1} B(\tau)}{\mathbb{E}\left(h_{t}\right)^{v}} \text { and } \quad L_{2} \stackrel{w}{\longrightarrow} \frac{\tau \sigma_{1} B(1)}{\mathbb{E}\left(h_{t}\right)^{v}}+\frac{\sigma_{2} \mathbb{E}\left(h_{t} \varepsilon_{t}^{2}\right)}{\mathbb{E}\left(h_{t}\right)^{v+1}}(W(\tau)-\tau W(1))
$$


by the invariance principle for dependent variables of [17].

$$
\left|L_{1}-L_{2}\right|=\frac{\sigma_{1}}{\mathbb{E}\left(h_{t}\right)^{v}}(B(\tau)-\tau B(1))-\frac{\sigma_{2} \mathbb{E}\left(h_{t} \varepsilon_{t}^{2}\right)}{\mathbb{E}\left(h_{t}\right)^{v+1}}(W(\tau)-\tau W(1))
$$

Finally, we have

$$
\tilde{e}_{n} \stackrel{w}{\longrightarrow} \frac{\sigma_{1} B^{0}(\tau)}{(\tau(1-\tau))^{\nu}}-\frac{\sigma_{2} \mathbb{E}\left(h_{t} \varepsilon_{t}^{2}\right)}{\mathbb{E}\left(h_{t}\right)(\tau(1-\tau))^{\nu}} W^{0}(\tau)
$$

where $B^{0}(\tau)$ and $W^{0}(\tau)$ denote standard brownian motions on [0,1] generated by $\left\{h_{t} \varepsilon_{t}^{2}\right\}_{t=1}^{\infty}$ and $\left\{h_{t}\right\}_{t=1}^{\infty}$ respectively.

\section{Volatility Change Point Detection for Exchange Rate Data}

We show an analysis of the United States Dollar (USD)-Ghana Cedi(GHS) daily exchange rate from 2008-2019 to illustrate the validity of the estimator. We consider the function of Equation (7) in our study.

$$
h_{t}\left(X_{t}, \theta\right) \rightarrow\left\{\begin{array}{l}
1 \text { for } \varepsilon_{t} \leq B \\
\frac{B}{\left|\varepsilon_{t}\right|} \text { for } \varepsilon_{t}>B
\end{array} \quad B>0\right.
$$

In this paper $B$ is chosen to be the 90th quantile of the residual data, $\varepsilon_{t}$.

The negative value of skewness and the small Jarque-Bera test value in Table 1 indicates the return data is not symmetric. The smaller p-value of the Arch test also indicates the presence of Arch effect.

Figure 1 shows the log return of the exchange rate data, the graph displays the typical "stylized fact" such as volatility clustering present in financial log-return series.

\subsection{ARMA Selection}

By observing the autocorrelation and partial autocorrelation and comparing the values of AIC and BIC, a more suitable order of $\mathrm{p}=1$ and $\mathrm{q}=1$ of the ARMA

Table 1. Statistical properties of return data.

\begin{tabular}{cc}
\hline \multicolumn{2}{c}{ Statistical Properties of Return Data } \\
\hline $\min$ & $-1.794944 \mathrm{e}-02$ \\
$\max$ & $1.183120 \mathrm{e}-02$ \\
median & $6.214180 \mathrm{e}-05$ \\
mean & $2.545361 \mathrm{e}-04$ \\
SE.mean & $2.722980 \mathrm{e}-05$ \\
var & $2.206591 \mathrm{e}-06$ \\
Arch Test & $\mathrm{p}$-value $=0.01$ \\
Jarque-Bera test $(\alpha=0.05)$ & $\mathrm{p}$-value $=2.2 \mathrm{e}-16$ \\
skewness & -2.189684 \\
kutosis & 46.1361 \\
\hline
\end{tabular}


USD-GHS RATES RETURN SERIES

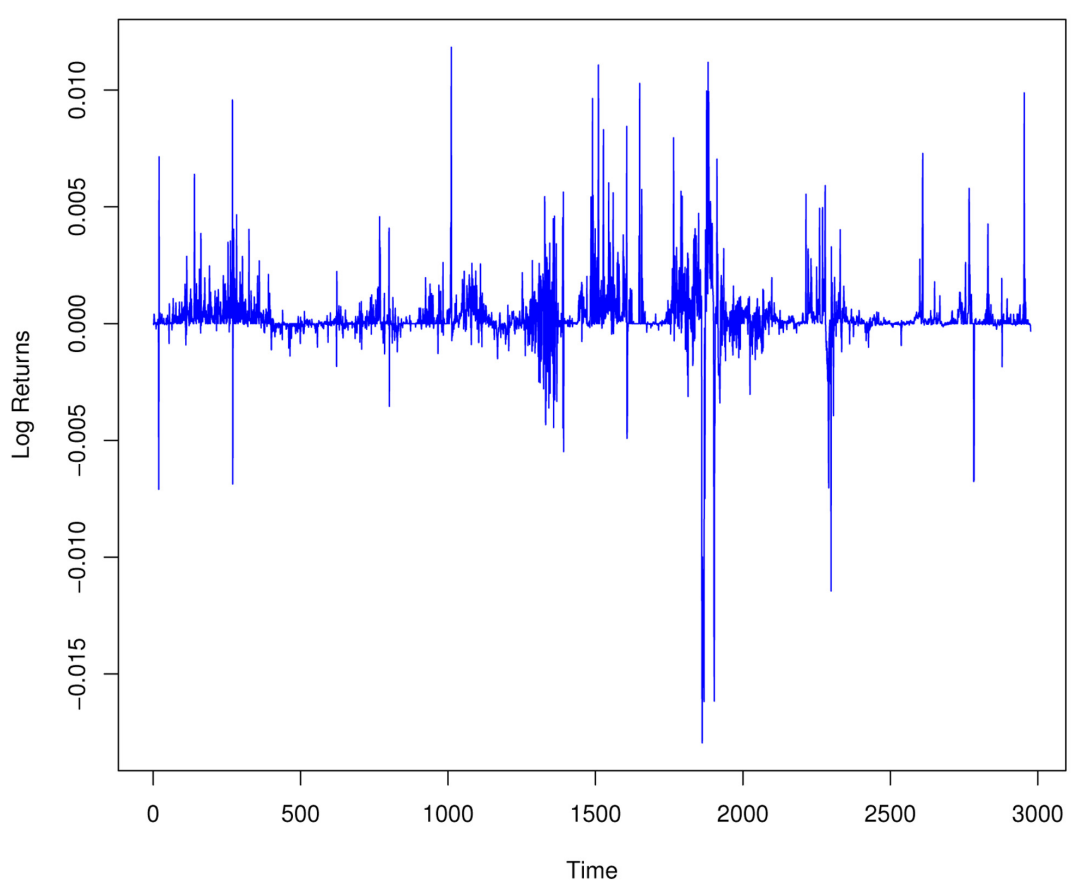

Figure 1. USD-GHS log return series from 2008-2019.

function was picked as shown in Equation (8):

$$
X_{t}=0.0000447210+0.8414551500 X_{t-1}-0.6086431547 \varepsilon_{t-1}+\varepsilon_{t}
$$

Table 2 shows the summary statistics from the residuals of the $\operatorname{ARMA}(1,1)$ model. The table shows a negatively skewed and highly heteroscedastic data.

\subsection{GARCH Results}

Based on the assumption of 5\% significance level, for the GARCH $(1,1)$ model, when the error term is skewed student- $t$ distribuion, the parameters for the $\operatorname{GARCH}(1,1)$ function is given as

$$
\sigma_{t}^{2}=0.0000000005+0.0515904234 \varepsilon_{t-1}^{2}+0.8974842734 \sigma_{t-1}^{2}
$$

From Table 3 and based on the assumption of 5\% significance level, for the $\operatorname{ARMA}(1,1)-\mathrm{GARCH}(1,1)$ model, all of the estimated parameters except omega are significant.

\subsection{Application of the Randomised Estimator to the USD-GHS Data}

We apply the randomised change point estimator to estimate the change point in the USD-GHS exchange rate data under the hypotheses;

$H_{0}$ : there is no change in variance.

$H_{a}$ : there is a change in variance.

In Figure 2 it was observed that there was a change in the variance of the exchange rate data at the 2213th data point with a test statistic value of 
Table 2. Statistical properties of residual data.

\begin{tabular}{cc}
\hline \multicolumn{2}{c}{ Statistical Properties of Residual Data } \\
\hline $\min$ & $-1.537934 \mathrm{e}-02$ \\
$\max$ & $1.080608 \mathrm{e}-02$ \\
mean & $8.489841 \mathrm{e}-05$ \\
SE.mean & $2.413864 \mathrm{e}-05$ \\
var & $1.734038 \mathrm{e}-06$ \\
Arch Test & p-value $=0.01$ \\
Jarque-Bera test $(\alpha=0.05)$ & p-value $=2.2 \mathrm{e}-16$ \\
skewness & -0.6262043 \\
kurtosis & 35.90743 \\
\hline
\end{tabular}

Table 3. Parameter estimation of the ARMA(1,1)-GARCH(1,1) model.

\begin{tabular}{ccccc}
\hline Variable & Estimate & Std. Error & t value & $\operatorname{Pr}(>|\mathrm{t}|)$ \\
\hline$\mu$ & 0.0000447210 & 0.0000064843 & 6.896791809 & 0.0000000000 \\
$\operatorname{AR}(1)$ & 0.8414551500 & 0.0191592710 & 43.918954357 & 0.0000000000 \\
$\mathrm{MA}(1)$ & -0.6086431547 & 0.0359025884 & -16.952626038 & 0.0000000000 \\
$\omega$ & 0.0000000005 & 0.0000000648 & 0.007050212 & 0.9943747915 \\
$\alpha_{1}$ & 0.0515904234 & 0.0044876976 & 11.495966890 & 0.0000000000 \\
$\beta_{1}$ & 0.8974842734 & 0.0061247224 & 146.534685788 & 0.0000000000 \\
\hline
\end{tabular}

upsilon $=0.2$

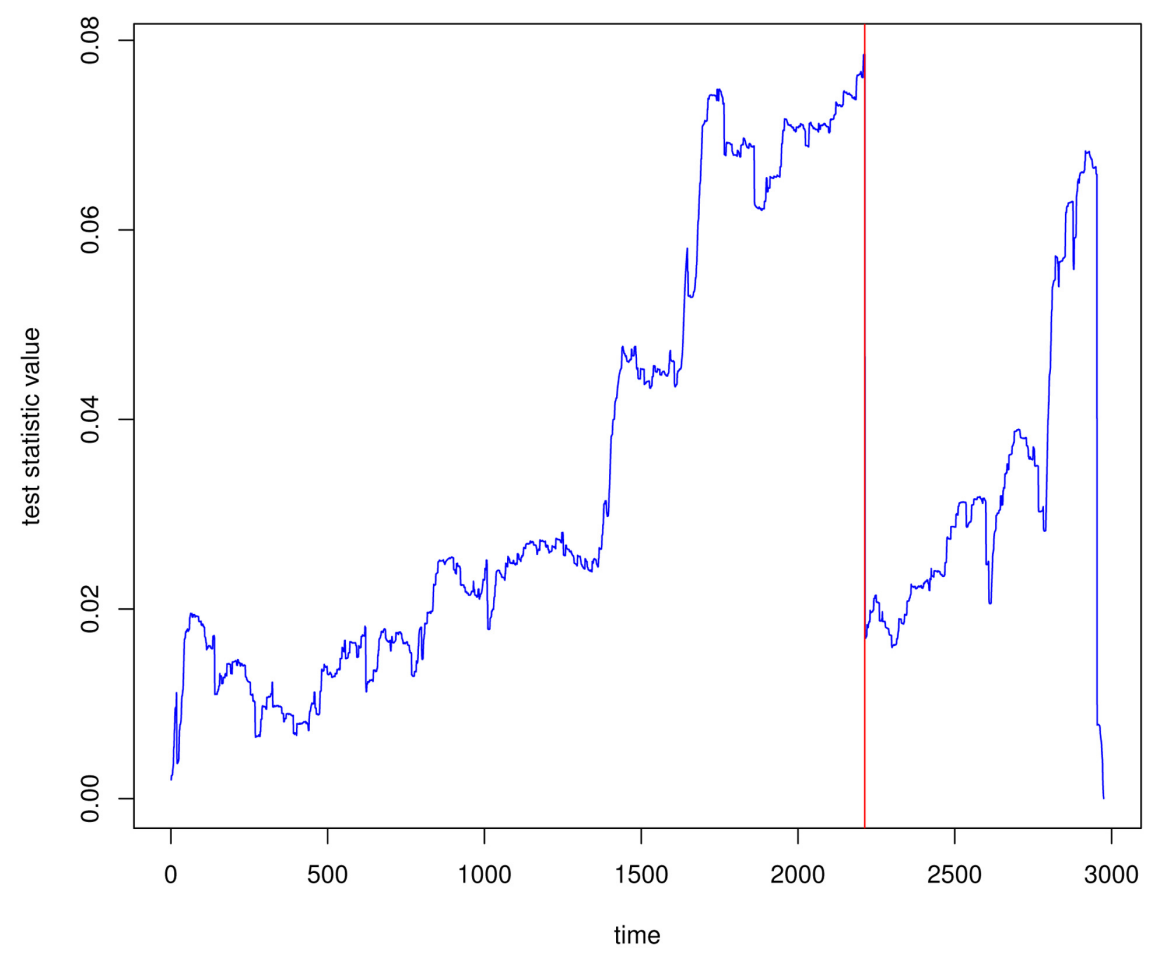

Figure 2. Randomised change point plot of the USD-GHS data indicating a change at the 2213th data point from a total of 2977 observations. 
$\tilde{e}=0.07859228$ when $v=0.2$. Consequently Figure 3 shows the point of change in the evolution of the daily exchange rate of the USD-GHS data.

\subsection{Data Analysis after the Change Point Estimator}

We analyze the mean and the variance of the exchange rate data after the application of randomised change point estimator. We present the mean and variance functions before and after the point of change.

\subsubsection{Before the Change Point}

The suitable mean model for the data before the change point is the ARMA(1,1)$\operatorname{GARCH}(1,1)$ and its parameters are given in Equations (10) and (11) when the error term is the skewed student $\mathrm{t}$-distribution.

$$
X_{t}=0.0000475035+0.8441708645 X_{t-1}-0.6457857840 \varepsilon_{t-1}+\varepsilon_{t}
$$

whilst the $\operatorname{GARCH}(1,1)$ model and is given as

$$
\sigma_{t}^{2}=0.0000000004+0.0536620794 \varepsilon_{t-1}^{2}+0.8958779068 \sigma_{t-1}^{2}
$$

\subsubsection{After the Change Point}

The suitable mean and variance model for the data after the point of change is the ARMA(1,1)-GARCH $(1,1)$ and its parameters are given in Equations (12) and (13) when the error term is the skewed student $t$-distribution.

\section{Change Point of USD-GHS Daily Exchange Rates}

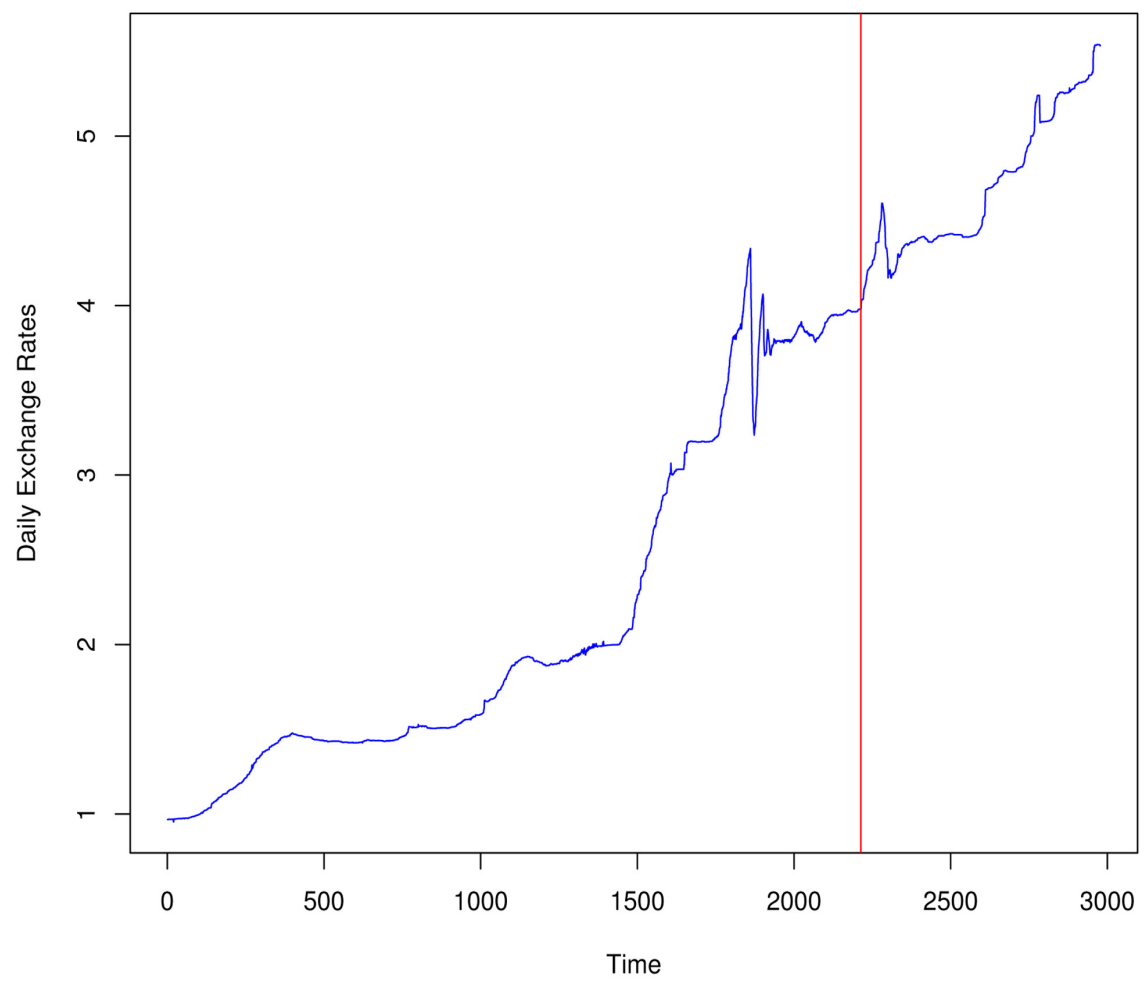

Figure 3. USD-GHS daily data from 2008-2019 and a change at the 2213th data point of a total of 2977 observations. 


$$
X_{t}=0.0000418753+0.5358615364 X_{t-1}-0.2512823195 \varepsilon_{t-1}+\varepsilon_{t}
$$

whilst the $\operatorname{GARCH}(1,1)$ model is given as

$$
\sigma_{t}^{2}=0.0000000004+0.0501059165 \varepsilon_{t-1}^{2}+0.8992657247 \sigma_{t-1}^{2}
$$

\subsection{Discussion}

The standard GARCH model was used as the measure of volatility. The persistence parameter $(\alpha+\beta)=0.94907$ of the sample remains relatively stable, though there seems to be an indication of some shifting parameters, the standard $\operatorname{GARCH}(1,1)$ appears to support the sample over the IGARCH model of [6]. The proposed randomised estimator identified a single possible change point value at $k=2213$ with a test statistic value, $\tilde{e}=0.07859228$ and a critical value of 3.31569 at $\alpha=0.05$ obtained from Equation (6) and corollary 1.3.1 of [7]. Hence, the test failed to reject the null hypothesis of no change in the variance of the exchange rate data. The persistence parameter before and after the change point were 0.94954 and 0.94937 respectively. The approximately $0 \%$ change in the unconditional variance between the two sub samples also supports the insignificant change in the variance structure of the exchange rate data.

The mean (ARMA) function of the data, however, gave a Root Mean Square Error (RMSE) of 0.00007699637 for a 10-period ahead forecast of the after change sample (Aft-ch) as against 0.00008529915 for the full sample. The result illustrates that change point test can be used as a reference point for historic data analysis.

\section{Conclusion}

In this paper, a randomised change point estimator for GARCH Models is presented. The limiting distribution of the estimator is derived as the sup of a standard Brownian bridge. Data analysis of the estimator was carried using United States Dollar (USD)-Ghana Cedi (GHS) daily exchange rate data. It was observed that the randomised estimator was able to detect and estimate a single change in variance of the data. The illustration shows that ignoring changes in data can lead to a false conclusion in statistical inference. For future studies we recommend other forms of the weight functions, $h_{t}$ be considered. It is worth mentioning that in this study a single change point test popularly referred to as At most one change (AMOC) was considered. We however, advocate for multiple changes (MOSUM) test via the binary segmentation technique.

\section{Acknowledgements}

The authors wish to thank the Pan African Univeristy Institute of Science, Technology and Innovation and the referees for their helpful suggestions.

\section{Funding Statement}

No external funding has been provided for this research. 


\section{Data Availability}

The authors used United States Dollar (USD)-Ghana cedi (GHS) daily exchange rate data obtained from the Ghana Stock Exchange (GSE).

\section{Conflicts of Interest}

The authors declare there is no conflict of interest.

\section{References}

[1] Awiakye-Marfo, G., Mung'atu, J. and O’Weke, P. (2020) Randomised Pseudolikelihood Ratio Change Point Estimator in Garch Models. Journal of Mathematics, 2020, Article ID: 6671515. https://doi.org/10.1155/2020/6671515

[2] Engle, R.F. (1982) Autoregressive Conditional Heteroscedasticity with Estimates of the Variance of United Kingdom Inflation. Econometrica: Journal of the Econometric Society, 50, 987-1007. https://doi.org/10.2307/1912773

[3] Bollerslev, T. (1986) Generalized Autoregressive Conditional Heteroskedasticity. Journal of Econometrics, 31, 307-327. https://doi.org/10.1016/0304-4076(86)90063-1

[4] Dieobold, F.X. (1986) Modeling the Persistence of Conditional Variances: A Comment. Econometric Reviews, 5, 51-56. https://doi.org/10.1080/07474938608800096

[5] Lamoureux, C.G. and Lastrapes, W.D. (1990) Persistence in Variance, Structural Change, and the Garch Model. Journal of Business \& Economic Statistics, 8, 225-234. https://doi.org/10.1080/07350015.1990.10509794

[6] Engle, R.F. and Bollerslev, T. (1986) Modelling the Persistence of Conditional Variances. Econometric Reviews, 5, 1-50. https://doi.org/10.1080/07474938608800095

[7] Csörgö, M. and Horváth, L. (1997) Limit Theorems in Change-Point Analysis. Volume 18, John Wiley \& Sons Inc., Amsterdam.

[8] Inclan, C. and Tiao, G.C. (1994) Use of Cumulative Sums of Squares for Retrospective Detection of Changes of Variance. Journal of the American Statistical Association, 89, 913-923. https://doi.org/10.1080/01621459.1994.10476824

[9] Kim, S., Cho, S. and Lee, S. (2000) On the Cusum Test for Parameter Changes in Garch $(1,1)$ Models. Communications in Statistics-Theory and Methods, 29, 445-462. https://doi.org/10.1080/03610920008832494

[10] Lee, S., Tokutsu, Y. and Maekawa, K. (2003) The Residual Cusum Test for the Constancy of Parameters in Garch $(1,1)$ Models. Working Paper, Seoul National University, Seoul.

[11] Irungu, I.W., Mwita, P.N. and Waititu, A.G. (2018) Limit Theory of Model Order Change-Point Estimator for Garch Models. Journal of Mathematical Finance, 8, 426. https://doi.org/10.4236/jmf.2018.82027

[12] Lee, J. and Lee, S. (2015) Parameter Change Test for Nonlinear Time Series Models with Garch Type Errors. Journal of the Korean Mathematical Society, 52, 503-522. https://doi.org/10.4134/JKMS.2015.52.3.503

[13] Zhou, J. and Liu, S.Y. (2009) Inference for Mean Change-Point in Infinite Variance AR (p) Process. Statistics \& Probability Letters, 79, 6-15. https://doi.org/10.1016/j.spl.2008.05.040

[14] Lee, S. and Oh, H. (2016) Parameter Change Test for Autoregressive Conditional Duration Models. Annals of the Institute of Statistical Mathematics, 68, 621-637. 
https://doi.org/10.1007/s10463-015-0541-x

[15] Oh, H. and Lee, S. (2019) Modified Residual CUSUM Test for Location-Scale Time Series Models with Heteroscedasticity. Annals of the Institute of Statistical Mathematics, 71, 1059-1091. https://doi.org/10.1007/s10463-018-0679-4

[16] Boussama, F. (1998) Ergodicité, mélange et estimation dans les modeles GARCH. PhD Thesis, Université Paris 7, Paris.

[17] McLeish, D.L. (1975) Invariance Principles for Dependent Variables. Zeitschrift für Wahrscheinlichkeitstheorie und verwandte Gebiete, 32, 165-178.

https://doi.org/10.1007/BF00532611 